\title{
RESISTÊNCIA DE DUAS ESPÉCIES DE BAMBU TRATADAS CONTRA FUNGOS XILÓFAGOS
}

Rogy Frigeri Tiburtino ${ }^{2}$, Juarez Benigno Paes ${ }^{3}$, Graziela Baptista Vidaurre ${ }^{4}$, Antonio Ludovico Beraldo ${ }^{5}$ e Marina Donária Chaves Arantes ${ }^{6}$

\begin{abstract}
RESUMO - O objetivo deste trabalho foi avaliar a resistência de bambus das espécies Bambusa vulgaris e Dendrocalamus giganteus após serem expostos a três métodos de tratamentos químicos preservativos, contra a ação dos fungos Postia placenta e Polyporus fumosus. Os métodos de tratamento empregados foram o de transpiração (diafragma íntegro e rompido), imersão prolongada e Boucherie modificado. As hastes de bambu foram transformadas em colmos de 2,0 m de comprimento e tratadas em solução de 1 ou $3 \%$ de ingredientes ativos (i.a.) de um produto comercial à base de cobre, cromo e boro (CCB). Nos métodos por transpiração e imersão prolongada, os colmos foram expostos nas soluções por períodos de 5, 10 ou 15 dias, enquanto no método de Boucherie modificado não houve segregação do tratamento entre tempos de tratamento. Para avaliar a eficiência dos tratamentos, foram empregados os fungos Postia placenta e Polyporus fumosus. A partir dos resultados, observou-se que, em média, em ambas as espécies de bambu tratadas e métodos empregados, a perda de massa das amostras de bambu, depois de submetidas ao ataque dos fungos, foi baixa, tendo variado de 2,44 a $14,26 \%$.
\end{abstract}

Palavras-chave: Dendrocalamus giganteus; Bambusa vulgaris; Tratamento preservativo

\section{RESISTANCE OF TWO TREATED BAMBOO SPECIES AGAINST XYLOPHAGOUS FUNGI}

\begin{abstract}
This work aimed to evaluate the resistance of bamboo species Bambusa vulgaris and Dendrocalamus giganteus, after being exposed to three methods of chemical preservative treatments against the action of Postia placenta and Polyporus fumosus fungi. The treatment methods employed were transpiration (integral and ruptured diaphragm), long-term immersion and Boucherie modified. The bamboo stems were transformed into culms of $2.0 \mathrm{~m}$ long and treated in a solution of 1 or 3\% active ingredient (a.i.) of a commercial product based on copper, chromium and boron (CCB). In the methods by transpiration and the long-term immersion, culms were exposed in solutions for periods of 5, 10 or 15 days, while in the modified Boucherie method there was no segregation of treatment between times of treatment. To assess the effectiveness of the treatments, Postia placenta and Polyporus fumosus fungi were employed. From the results obtained it was observed that, on average, for both species of bamboo and tested methods, the mass loss of samples of bamboo, after undergoing the fungal attack, was low, varying from 2.44 to $14.26 \%$.
\end{abstract}

Keywords: Dendrocalamus giganteus; Bambusa vulgaris; Preservative treatment.

\footnotetext{
${ }^{1}$ Recebido em 28.01.2013 aceito para publicação em 24.03.2015

${ }^{2}$ Universidade Federal do Espírito Santo, Mestrado em Ciências Florestais, Jerônimo Monteiro, Espírito Santo, Brasil. E-mail: <rogyfrigeri@hotmail.com>.

${ }^{3}$ Universidade Federal do Espírito Santo, Departamento de Ciências Florestais e da Madeira, Jerônimo Monteiro, Espírito Santo, Brasil. E-mail: <jbp2@uol.com.br>.

${ }^{4}$ Universidade Federal do Espírito Santo, Departamento de Ciências Florestais e da Madeira, Jerônimo Monteiro, Espírito Santo, Brasil. E-mail: <grazividaurre@gmail.com>.

${ }^{5}$ Universidade Estadual de Campinas, Faculdade de Engenharia Agrícola, Campinas, São Paulo, Brasil. E-mail: <beraldo@feagri.unicamp.br>.

${ }^{6}$ Universidade Federal do Espírito Santo, Departamento de Ciências Florestais e da Madeira, Jerônimo Monteiro, Espírito Santo, Brasil. E-mail:<mdonariac@hotmail.com>.
} 


\section{INTRODUÇ̃̃O}

Após vários anos de exploração sem controle, as espécies arbóreas nativas de boa durabilidade natural se tornaram escassas e de custo elevado em diversas regiões brasileiras (FARIAS SOBRINHO et al., 2005). Tal fato ocorreu, principalmente, em razão de sua intensa utilização em construções, como em cercas, estruturas para telhados, móveis, currais e galpões. Por causa dessa situação, a procura por matérias-primas alternativas que sejam de fontes renováveis e que tenham características físicas e mecânicas semelhantes às da madeira é cada vez maior (PAES et al., 2009), e o bambu é um exemplo de material alternativo.

O bambu é um compósito natural lignocelulósico de baixa massa, alta resistência mecânica, fácil manuseio, rápido crescimento e de grande disponibilidade, o que torna fácil a sua obtenção, podendo ser utilizado no paisagismo rural e como elemento estrutural à medida que atinge a idade adulta (BERALDO; RIVERO, 2003). No entanto, por causa da sua constituição fibrosa e rica em materiais nutritivos, principalmente amido, os bambus são suscetíveis ao ataque de fungos e insetos xilófagos (BERALDO et al., 2012).

De acordo com Mohanan (1997), colmos de bambus armazenados, sob determinadas condições, desenvolvem fungos de coloração escura com tons de marrom a preto, com manchas causadas pelos Ascomycetes; o ataque ocorre, em geral, superficialmente e pode ser removido ao ser escovado ou raspado. Contudo, as manchas podem penetrar profundamente no colmo, depreciando o seu valor comercial.

Doenças do colmo são facilmente identificáveis pela sua forma peculiar, como a ferrugem e outros fungos. $\mathrm{Na}$ China, têm sido encontradas 22 espécies de fungos decompositores pertencentes a 16 gêneros (HAOJIE et al., 1998).

Beraldo et al. (2012) isolaram 10 filamentos de fungos obtidos de amostras de Dendrocalamus giganteus expostas às condições do ambiente, sendo identificados como pertencentes aos gêneros Arthrinum, Fusarium, Acremonium e Trichoderma. Isso indica que dificilmente o bambu teria ataque de um tipo de fungo isoladamente.

Há poucos trabalhos científicos direcionados para o conhecimento da resistência do bambu, tratado ou não, ao ataque de fungos deterioradores. Diante dessa constatação, esta pesquisa teve como objetivo avaliar a resistência de bambus das espécies Dendrocalamus giganteus Munro e Bambusa vulgaris Schrad. ex J.C. Wendl. após serem expostos a três métodos de tratamentos químicos preservativos contra a ação dos fungos Postia placenta (Fr.) M. J. Lars. \& Lomb. e Polyporus fumosus Pers. ex Fries.

\section{MATERIAL E MÉTODOS}

\subsection{Tratamentos preservativos empregados}

Hastes de bambu das espécies Dendrocalamus giganteus e Bambusa vulgaris foram coletadas de touceiras, próximas aos Municípios de Alegre e Jerônimo Monteiro, Sul do Estado do Espírito Santo. O bambu Dendrocalamus giganteus foi escolhido para o trabalho em razão de suas propriedades físico-mecânicas, espessura de parede e diâmetro dos colmos. Já o Bambusa vulgaris é o bambu de maior ocorrência no território brasileiro.

As hastes foram transformadas em colmos de 2,0 $m$ de comprimento e tratadas em soluções de 1 ou $3 \%$ de ingredientes ativos (i.a.) de um produto comercial à base de cobre, cromo e boro (CCB), por meio de três métodos preservativos: transpiração, imersão prolongada e Boucherie modificado. O produto empregado, segundo a Norma Brasileira Regulamentadora - NBR 9480, da Associação Brasileira de Normas Técnicas (ABNT, 2009), deve conter $63,5 \%$ de cromo $\left(\mathrm{CrO}_{3}\right), 26,0 \%$ de cobre $(\mathrm{CuO})$ e $10,5 \%$ de boro (B elemento).

No método de transpiração, os colmos ficaram submersos na solução preservativa por 5,10 ou 15 dias, e cada tempo representou um tratamento, conforme metodologia descrita por Paes (1991), Santos e Lopez (1998), Farias Sobrinho et al. (2005) e Paes et al. (2005). Os colmos foram dispostos verticalmente em tambores de $200 \mathrm{~L}$, ficando submersos $(0,5 \mathrm{~m}$ da base $)$ na solução preservativa. O tratamento foi realizado sob duas situações (diafragma íntegro e diafragma rompido). Essa iniciativa foi tomada com base nas literaturas, mencionadas por Liese (1985) e Hui e Yang (2010), que descrevem, entre outras características, a anatomia dos nós, particularmente dos diafragmas, os quais podem obstruir a movimentação da solução preservativa.

No método de imersão prolongada, os colmos foram distribuídos em cochos (2,10 m de comprimento $\mathrm{x} 0,44$ $\mathrm{m}$ de largura $\mathrm{x} 0,56 \mathrm{~m}$ de profundidade) confeccionados com compensado resistente à umidade, tendo sido

Revista Árvore, Viçosa-MG, v.39, n.3, p.567-574, 2015

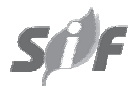


forrados internamente com lona plástica. Os diafragmas foram rompidos, para auxiliar na absorção da solução preservativa e dificultar a flutuação deles nas soluções de tratamento. Os bambus ficaram submersos na solução preservativa por 5,10 ou 15 dias

No método de Boucherie modificado, foi utilizada uma pressão manométrica de $0,1 \mathrm{MPa}$, para o tratamento dos colmos. A pressão foi exercida pela alimentação de ar comprimido para o interior de uma autoclave, onde estava a solução química. A autoclave se conectava aos colmos de bambu, por meio de uma tubulação de PVC. A tubulação era conectada à válvula de escape localizada na autoclave e a solução era liberada, permitindo que preenchesse a tubulação e, por meio de quatro válvulas na tubulação, a solução preenchia o primeiro entrenó do bambu e era forçada através dos colmos. Nesse método, os tratamentos não foram divididos em tempos, conforme nos demais, e sim pelo tempo de escoamento da solução pela extremidade oposta à da aplicação de pressão.

\subsection{Ensaios de resistência a fungos xilófagos}

No ensaio com fungos, frascos de $600 \mathrm{~mL}$ foram preenchidos com $300 \mathrm{~g}$ de solo de $\mathrm{pH}$ 5,6 e capacidade de retenção de água de $28 \%$, conforme recomendado pela "American Society for Testing and Materials" ASTM D-1413 (2005a). Após o preenchimento, o solo foi umedecido com $86 \mathrm{~mL}$ de água destilada e adicionados dois alimentadores de madeira de Pinus sp., para o desenvolvimento dos fungos, conforme citado na norma técnica empregada.

Os corpos de prova, com comprimento e largura de 2,0 x 2,0 cm e espessura da parede do colmo, foram secos em estufa a $103 \pm 2{ }^{\circ} \mathrm{C}$, por um período de 48 $\mathrm{h}$; a massa de cada amostra foi determinada conforme recomendado pela ASTM D-1413 (2005a); e os valores obtidos foram utilizados no cálculo da perda de massa do bambu causada pelos fungos utilizados.

Conforme recomendações daASTMD-1413 (2005a), os frascos contendo solo umedecido foram esterilizados em autoclave a $121 \pm 2{ }^{\circ} \mathrm{C}$, por $50 \mathrm{~min}$, e climatizados. Em cada recipiente, foram inoculados sobre os alimentadores fragmentos obtidos de culturas puras dos fungos Postia placenta (Fr.) M. J. Lars. \& Lomb. e Polyporus fumosus Pers. ex Fries. A espécie de fungo Postia placenta causa deterioração da celulose na madeira, já a espécie Polyporus fumosus consome as hemiceluloses e a lignina. Com o ataque desses fungos ocorre diminuição na perda de massa e nas propriedades mecânicas da madeira. Por esses motivos, essas duas espécies de fungos foram escolhidas para serem empregadas neste trabalho, com o intuito de analisar o comportamento do bambu tratado contra a ação desses fungos.

Após o período de 15 dias ocorreram o desenvolvimento do fungo e a colonização do solo, tendo sido adicionados dois corpos de prova por frasco, em um total de duas repetições, por combinação de fungos, tratamento preservativo, tempo de tratamento (transpiração e imersão), concentração das soluções e espécie de bambu.

Os frascos para o desenvolvimento do ensaio biológico foram mantidos em sala climatizada ( $25 \pm$ $2{ }^{\circ} \mathrm{C}$ e $75 \pm 5 \%$ de umidade relativa), por 98 dias. Ao final do ensaio, foram realizadas a limpeza dos corpos de prova e a sua secagem em estufa. A perda de massa foi avaliada e classificada conforme o recomendado pela ASTM D-2017 (2005b).

\subsection{Análises estatísticas dos resultados}

Para avaliação estatística da perda de massa pela ação dos fungos, foi empregado o delineamento inteiramente casualizado com arranjo fatorial. Para realização do ensaio, foram utilizadas amostras retiradas a $0,5 \mathrm{~m}$ de altura da base de cada colmo, sendo esse local considerado a região de afloramento para peças instaladas no solo.

Foram empregadas duas repetições para cada método e condição de tratamento na avaliação estatística dos ensaios com fungos. Para a retirada dos corpos de prova, foi fixada uma posição no colmo $(0,5 \mathrm{~m}$ de altura da base), sendo os resultados analisados em razão do tempo de tratamento (três níveis), concentração das soluções preservativas (dois níveis) e tipos de fungos (dois níveis).

Os dados, em porcentagem de perda de massa, foram transformados em arcosseno [raiz quadrada (valor em porcentagem/100)], conforme sugerido por Steel e Torrie (1980). Essas transformações foram necessárias por causa da necessidade de homogeneizar as variâncias. Foi empregado o teste de Tukey para comparação das médias a 5\% de significância, para os fatores e interações detectadas como significativos pelo teste de F.

Revista Árvore, Viçosa-MG, v.39, n.3, p.567-574, 2015 


\section{RESULTADOS}

Nas Tabelas 1 e 2 constam as médias das perdas de massa (\%) das amostras das duas espécies de bambu tratadas, causadas pela ação dos fungos Postia placenta e Polyporus fumosus, respectivamente, e a classificação da resistência dos bambus ao ataque desses fungos, de acordo com a ASTM D 2017 (2005b), que classifica como muito resistente material lignocelulósico com perdas de massa de 0 a $10 \%$, resistente de 11 a $24 \%$, resistência moderada de 25 a 44\% e não resistente quando igual ou superior a 45\%. Notam-se, em ambas as espécies de bambu empregadas, que as perdas de massa foram baixas, tendo variado de $2,60 \pm 1,00$ a $14,26 \pm 4,45 \%$ (Postia placenta) e de 2,57 $\pm 0,03$ a 18,80 $\pm 2,81 \%$ (Polyporus fumosus), sendo classificadas, em sua maioria, como muito resistente (ASTM D 2017, 2005b).

Os valores que deram origem às Tabelas 1 e 2 foram analisados estatisticamente, tendo sido, pelo método de transpiração (diafragma íntegro), obtido diferenças significativas entre os parâmetros tempo de tratamento, em ambas as espécies de bambu, fungos e concentração das soluções, para o B. vulgaris. Para o método com diafragma rompido, foram observadas diferenças significativas entre as concentrações (D. giganteus) e para a interação tempo e concentração na espécie B. vulgaris. As médias dos parâmetros e da interação (após o desdobramento) foram comparadas pelo teste de Tukey a $5 \%$ de significância e consta na Tabela 3 .

Com o método de imersão prolongada, foi observado efeito significativo apenas entre a interação concentração e o tempo de tratamento da espécie B. vulgaris, enquanto com o método de Boucherie modificado obtiveramse efeitos significativos dos fatores fungos e concentração da solução de tratamento, também de B. vulgaris. Nesses casos, foi realizada a comparação entre as médias das perdas de massa. Na Tabela 4 constam

Tabela 1 - Valores médios da perda de massa e classificação dos bambus quanto à resistência ao ataque do fungo Postia placenta pelos métodos, tempo de tratamento e concentração da solução preservante.

Table 1 - Average values of mass loss and classification of bamboos as for the resistance to attack of Postia placenta fungus according to methods, treatment time and preservative solution concentration.

\begin{tabular}{|c|c|c|c|c|}
\hline \multirow{3}{*}{ Métodos } & \multirow{3}{*}{ Espécies } & \multirow{3}{*}{$\begin{array}{c}\text { Tempo de tratamento } \\
\text { (Dias) }\end{array}$} & \multicolumn{2}{|c|}{ Perda de massa (\%) } \\
\hline & & & \multicolumn{2}{|c|}{ Concentração da solução (\%) } \\
\hline & & & 1 & 3 \\
\hline Transpiração & D. giganteus & 5 & $5,50 \pm 2,06(\mathrm{MR})$ & $6,80 \pm 2,84(\mathrm{MR})$ \\
\hline \multirow[t]{5}{*}{ (Diafragma Íntegro) } & & 10 & $7,88 \pm 4,00(\mathrm{MR})$ & $5,38 \pm 1,27(\mathrm{MR})$ \\
\hline & & 15 & $3,82 \pm 0,50(\mathrm{MR})$ & $3,03 \pm 0,70(\mathrm{MR})$ \\
\hline & B. vulgaris & 5 & $10,00 \pm 2,11(\mathrm{MR})$ & $6,00 \pm 1,83(\mathrm{MR})$ \\
\hline & & 10 & $5,27 \pm 2,22(\mathrm{MR})$ & $6,21 \pm 3,00(\mathrm{MR})$ \\
\hline & & 15 & $4,58 \pm 1,69(\mathrm{MR})$ & $2,44 \pm 0,04(\mathrm{MR})$ \\
\hline Transpiração & D. giganteus & 5 & $6,50 \pm 3,40(\mathrm{MR})$ & $7,45 \pm 0,95(\mathrm{MR})$ \\
\hline \multirow[t]{5}{*}{ (Diafragma Rompido) } & & 10 & $5,90 \pm 0,89(\mathrm{MR})$ & $3,01 \pm 0,67(\mathrm{MR})$ \\
\hline & & 15 & $6,63 \pm 2,84(\mathrm{MR})$ & $2,60 \pm 1,00(\mathrm{MR})$ \\
\hline & B. vulgaris & 5 & $8,25 \pm 0,70(\mathrm{MR})$ & $6,35 \pm 1,13(\mathrm{MR})$ \\
\hline & & 10 & $4,50 \pm 0,27(\mathrm{MR})$ & $3,08 \pm 0,60(\mathrm{MR})$ \\
\hline & & 15 & $3,22 \pm 0,51(\mathrm{MR})$ & $4,95 \pm 0,97(\mathrm{MR})$ \\
\hline \multirow[t]{6}{*}{ Imersão Prolongada } & D. giganteus & 5 & $5,05 \pm 1,29(\mathrm{MR})$ & $6,90 \pm 1,08(\mathrm{MR})$ \\
\hline & & 10 & $6,50 \pm 0,37(\mathrm{MR})$ & $8,50 \pm 6,82(\mathrm{MR})$ \\
\hline & & 15 & $6,18 \pm 1,47(\mathrm{MR})$ & $11,73 \pm 10,60(\mathrm{R})$ \\
\hline & B. vulgaris & 5 & $10,34 \pm 0,41(\mathrm{R})$ & $9,01 \pm 3,62(\mathrm{MR})$ \\
\hline & & 10 & $9,00 \pm 1,48(\mathrm{MR})$ & $8,80 \pm 1,06(\mathrm{MR})$ \\
\hline & & 15 & $6,31 \pm 1,28(\mathrm{MR})$ & $3,10 \pm 0,22(\mathrm{MR})$ \\
\hline \multirow[t]{2}{*}{ Boucherie Modificado } & D. giganteus & - & $12,35 \pm 1,52(\mathrm{R})$ & $6,42 \pm 3,55(\mathrm{MR})$ \\
\hline & B. vulgaris & - & $4,82 \pm 0,40(\mathrm{MR})$ & $14,26 \pm 4,45(\mathrm{R})$ \\
\hline \multirow[t]{2}{*}{ Controle } & D. giganteus & - & $10,83 \pm 2,24(\mathrm{R})$ & \\
\hline & B. vulgaris & - & $8,33 \pm 0,93(\mathrm{MR})$ & \\
\hline
\end{tabular}

$\mathrm{MR}=$ Muito Resistente; e R = Resistente (ASTM - D 2017, 2005b).

Revista Árvore, Viçosa-MG, v.39, n.3, p.567-574, 2015 
Tabela 2 - Valores médios de perdas de massa e classificação dos bambus quanto à resistência ao ataque do fungo Polyporus fumosus para os métodos, tempo de tratamento e concentração da solução preservante.

Table 2 - Average values of mass loss and classification of bamboos as for the resistance to attack of Polyporus fumosus fungus according to methods, treatment time and preservative solution concentration.

\begin{tabular}{|c|c|c|c|c|}
\hline \multirow{3}{*}{ Métodos } & \multirow{3}{*}{ Espécies } & \multirow{3}{*}{$\begin{array}{c}\text { Tempo de tratamento } \\
\text { (Dias) }\end{array}$} & \multicolumn{2}{|c|}{ Perda de massa $(\%)$} \\
\hline & & & \multicolumn{2}{|c|}{ Concentração da solução (\%) } \\
\hline & & & 1 & 3 \\
\hline Transpiração & D. giganteus & 5 & $6,30 \pm 4,40(\mathrm{MR})$ & $4,00 \pm 2,42(\mathrm{MR})$ \\
\hline \multirow[t]{5}{*}{ (Diafragma Íntegro) } & & 10 & $6,70 \pm 1,27(\mathrm{MR})$ & $6,15 \pm 1,28(\mathrm{MR})$ \\
\hline & & 15 & $3,86 \pm 0,15(\mathrm{MR})$ & $3,48 \pm 0,46(\mathrm{MR})$ \\
\hline & B. vulgaris & 5 & $12,57 \pm 3,73(\mathrm{R})$ & $5,18 \pm 1,11(\mathrm{MR})$ \\
\hline & & 10 & $12,15 \pm 4,46(\mathrm{R})$ & $6,36 \pm 0,32(\mathrm{MR})$ \\
\hline & & 15 & $8,38 \pm 0,81(\mathrm{MR})$ & $6,10 \pm 0,73(\mathrm{MR})$ \\
\hline Transpiração & D. giganteus & 5 & $5,16 \pm 2,14(\mathrm{MR})$ & $5,03 \pm 1,00(\mathrm{MR})$ \\
\hline \multirow[t]{5}{*}{ (Diafragma Rompido) } & & 10 & $4,07 \pm 2,35(\mathrm{MR})$ & $2,91 \pm 0,03(\mathrm{MR})$ \\
\hline & & 15 & $7,18 \pm 3,01(\mathrm{MR})$ & $2,57 \pm 1,41(\mathrm{MR})$ \\
\hline & B. vulgaris & 5 & $5,38 \pm 1,03(\mathrm{MR})$ & $6,42 \pm 0,27(\mathrm{MR})$ \\
\hline & & 10 & $4,71 \pm 0,86(\mathrm{MR})$ & $3,00 \pm 0,11(\mathrm{MR})$ \\
\hline & & 15 & $3,30 \pm 0,11(\mathrm{MR})$ & $4,92 \pm 1,80(\mathrm{MR})$ \\
\hline \multirow[t]{6}{*}{ Imersão Prolongada } & D. giganteus & 5 & $5,45 \pm 0,11(\mathrm{MR})$ & $6,66 \pm 0,48(\mathrm{MR})$ \\
\hline & & 10 & $6,14 \pm 0,48(\mathrm{MR})$ & $5,66 \pm 0,75(\mathrm{MR})$ \\
\hline & & 15 & $7,16 \pm 1,83(\mathrm{MR})$ & $6,66 \pm 0,46(\mathrm{MR})$ \\
\hline & B. vulgaris & 5 & $10,02 \pm 2,22(\mathrm{R})$ & $12,47 \pm 2,30(\mathrm{R})$ \\
\hline & & 10 & $10,10 \pm 1,42(\mathrm{R})$ & $10,00 \pm 1,07(\mathrm{MR})$ \\
\hline & & 15 & $7,37 \pm 1,65(\mathrm{MR})$ & $3,87 \pm 0,58(\mathrm{MR})$ \\
\hline \multirow[t]{2}{*}{ Boucherie modificado } & D. giganteus & - & $10,58 \pm 1,67(\mathrm{R})$ & $8,15 \pm 3,33(\mathrm{MR})$ \\
\hline & B. vulgaris & - & $11,07 \pm 3,00(\mathrm{R})$ & $18,80 \pm 2,81(\mathrm{R})$ \\
\hline \multirow[t]{2}{*}{ Controle } & D. giganteus & - & $8,35 \pm 0,61(\mathrm{MR})$ & \\
\hline & B. vulgaris & - & $11,24 \pm 0,06(\mathrm{R})$ & \\
\hline
\end{tabular}

$\mathrm{MR}=$ Muito Resistente; e R = Resistente (ASTM - D 2017, 2005b).

Tabela 3 - Perdas de massa média de tempo de tratamento, fungos e concentração da solução das espécies de bambu $D$. giganteus e $B$. vulgaris tratadas pelo método de transpiração (diafragma íntegro ou rompido).

Table 3 - Average of mass losses for treatment time, fungi and solution concentration of D. giganteus and B. vulgaris bamboo species treated by transpiration method (integral or ruptured diaphragm).

\begin{tabular}{|c|c|c|c|}
\hline \multicolumn{4}{|c|}{ Transpiração (Diafragma Íntegro) } \\
\hline \multirow{2}{*}{ Tempo de tratamento (Dias) } & \multicolumn{3}{|c|}{ Perda de massa (\%) } \\
\hline & D. giganteus & \multicolumn{2}{|c|}{ B. vulgaris } \\
\hline 5 & $5,65 \mathrm{ab}$ & \multicolumn{2}{|c|}{8,44 a } \\
\hline 10 & 6,53 a & \multicolumn{2}{|c|}{$7,50 \mathrm{ab}$} \\
\hline 15 & $3,55 \mathrm{~b}$ & \multicolumn{2}{|c|}{$5,40 \mathrm{~b}$} \\
\hline \multicolumn{4}{|c|}{ B. vulgaris } \\
\hline Fungos & Perda de massa $(\%)$ & Concentração (\%) & Perda de massa $(\%)$ \\
\hline Postia placenta & $5,75 \mathrm{~b}$ & 1 & 8,83 a \\
\hline P. fumosus & 8,44 a & 3 & $5,38 \mathrm{~b}$ \\
\hline \multicolumn{4}{|c|}{ Transpiração (Diafragma Rompido) } \\
\hline \multicolumn{4}{|c|}{ D. giganteus } \\
\hline \multicolumn{2}{|c|}{ Concentração (\%) } & \multicolumn{2}{|c|}{ Perda de massa $(\%)$} \\
\hline \multicolumn{2}{|c|}{$\begin{array}{l}1 \\
3\end{array}$} & \multicolumn{2}{|c|}{$\begin{array}{l}5,91 \mathrm{a} \\
3,93 \mathrm{~b}\end{array}$} \\
\hline \multicolumn{4}{|c|}{ B. vulgaris } \\
\hline \multirow[t]{2}{*}{ Concentração (\%) } & \multicolumn{3}{|c|}{ Tempo tratamento (Dias)/Perda de massa (\%) } \\
\hline & 5 & 10 & 15 \\
\hline 1 & $6,81 \mathrm{Aa}$ & $4,61 \mathrm{Ba}$ & $3,26 \mathrm{Bb}$ \\
\hline 3 & $6,36 \mathrm{Aa}$ & $3,04 \mathrm{Bb}$ & 4,94 $\mathrm{Aa}$ \\
\hline
\end{tabular}

As médias seguidas da mesma letra, maiúscula na horizontal, ou minúscula na vertical, para cada parâmetro analisado, não diferem estatisticamente pelo teste de Tukey $(\mathrm{p}>0,05)$. 
Tabela 4 - Perda de massa média da concentração e tempo de tratamento da espécie B. vulgaris tratada pelo método de imersão prolongada.

Table 4 - Average of mass loss for concentration and treatment time of $\boldsymbol{B}$. vulgaris bamboo specie treated by long-term immersion.

\begin{tabular}{|c|c|c|c|}
\hline \multicolumn{4}{|c|}{ Imersão prolongada } \\
\hline \multicolumn{4}{|c|}{ Bambusa vulgaris } \\
\hline \multirow{2}{*}{ Concentração (\%) } & \multicolumn{3}{|c|}{ Tempo de tratamento (Dias)/Perda de massa (\%) } \\
\hline & 5 & 10 & 15 \\
\hline 1 & $10,18 \mathrm{Aa}$ & $9,55 \mathrm{ABa}$ & $6,84 \mathrm{Ba}$ \\
\hline 3 & $10,74 \mathrm{Aa}$ & $9,40 \quad \mathrm{Aa}$ & $3,49 \mathrm{Bb}$ \\
\hline
\end{tabular}

As médias seguidas da mesma letra, maiúscula na horizontal ou minúscula na vertical, de cada parâmetro analisado não diferem estatisticamente, pelo teste de Tukey $(\mathrm{p}>0,05)$.

as comparações do tratamento por imersão prolongada e, na Tabela 5, do método de Boucherie modificado.

\section{DISCUSSÃO}

Observa-se, na Tabela 1, que os corpos de prova ofereceram muita resistência (MR) ao ataque do fungo Postia placenta em praticamente todos os tratamentos, inclusive no controle, em que apenas cinco médias foram classificadas como resistentes (R). Os bambus tratados pelos métodos de imersão prolongada e Boucherie modificado obtiveram médias mais elevadas de perda de massa para cada espécie e concentração da solução preservativa.

Como consta na Tabela 2, a maioria das médias de perdas de massa indica que as amostras obtidas de ambas as espécies de bambu e tratadas pelos métodos de tratamento empregados foram classificadas como muito resistentes à ação do fungo Polyporus fumosus. As duas espécies tratadas pelo método de Boucherie modificado e o $B$. vulgaris submetido à imersão prolongada atingiram perdas de massa mais elevadas.

Em todos os métodos de tratamento empregados no fungo Polyporus fumosus, foi observado, na espécie B. vulgaris, que as perdas variaram de 3,00 a $18,80 \%$ e, em D. giganteus, de 2,57 a $10,58 \%$. Assim como se verificou na avaliação do fungo $P$. placenta, os resultados de perda de massa após o ataque do fungo $P$. fumosus evidenciaram que as amostras tratadas por transpiração com diafragma rompido, nas duas espécies, exibiram menores perdas de massa.

Suprapti (2010) expôs amostras das espécies $B$. vulgaris, sem a retirada das camadas externa e interna, à ação dos fungos Postia placenta e Polyporus sp.,
Tabela 5 - Perda de massa média da espécie B. vulgaris dos fungos e concentrações das soluções, pelo método de Boucherie modificado.

Table 5 -Average of mass loss for $\boldsymbol{B}$. vulgaris bamboo specie according to fungi and solution concentrations, using the Boucherie modified method.

\begin{tabular}{cc}
\hline \multicolumn{2}{c}{ Boucherie modificado } \\
\hline \multicolumn{2}{c}{ Bambusa vulgaris } \\
\hline Fungos & Perda de massa (\%) \\
\hline Postia placenta & $9,54 \mathrm{~b}$ \\
Polyporus fumosus & $14,94 \mathrm{a}$ \\
\hline Concentração (\%) & Perda de massa (\%) \\
\hline 1 & $7,95 \mathrm{~b}$ \\
3 & $16,53 \mathrm{a}$ \\
\hline
\end{tabular}

As médias seguidas da mesma letra em cada parâmetro não diferem estatisticamente, pelo teste de Tukey $(p>0,05)$.

para determinar suas resistências naturais, sendo a avaliação realizada em três posições do colmo (base, meio e topo). O trabalho evidenciou que a espécie de bambu foi mais resistente ao ataque do P. placenta em comparação com o Polyporus sp.

Observa-se, na Tabela 3, que nas amostras do tratamento com diafragma íntegro para os tempos de 5 e 10 dias ocorreram as perdas mais elevadas nas duas espécies. Ainda analisando o mesmo tratamento, verificou-se que o fungo Polyporus fumosus e a concentração de $1 \%$ de i.a. exibiram os valores mais elevados de perdas de massa.

Ao analisar, na Tabela 3, as amostras tratadas pelo método de transpiração com diafragma rompido, notou-se que o parâmetro de concentração da solução para o D. giganteus se comportou de forma semelhante ao 
do tratamento com diafragma íntegro, ou seja, as amostras submetidas ao tratamento com $3 \%$ de i.a. proporcionaram, em média, menor perda de massa, aproximadamente $21 \%$ menor.

As comparações das médias do B. vulgaris, expostas na Tabela 3, obtidas pelo método de transpiração com diafragma rompido, evidenciam que, na concentração de $1 \%$ de i.a., o tempo de cinco dias forneceu maior valor de perda de massa, porém na concentração de $3 \%$ os tempos de 5 e 15 dias exibiram resultados estatisticamente semelhantes. E, ao analisar o tempo de 15 dias, a média de perda de massa foi maior em $3 \%$ de i.a.

Para o método de imersão prolongada, a análise de variância não indicou resultados estatisticamente significativos para a perda de massa nas amostras da espécie D. giganteus. Em B. vulgaris, houve diferença na interação entre tempo e concentração. A interação foi desdobrada (Tabela 4), observando-se nos tempos de 5 e 10 dias semelhança estatística entre as médias de perdas de massa, enquanto no tempo de 15 dias os bambus tratados com solução de $1 \%$ de i.a. tiveram as perdas de massa mais elevadas.

No método de Boucherie modificado, observou-se que os valores de massa perdida do B. vulgaris na concentração com $3 \%$ de i.a. e no fungo Polyporus fumosus foram mais acentuados (Tabela 5). Pelas análises estatísticas, foi verificado que as perdas de massa das amostras da espécie $D$. giganteus não apresentaram diferenças estatísticas.

De modo geral, em muitos casos, nos quatros tratamentos empregados os melhores resultados de resistência ao ataque dos fungos foram observados em amostras tratadas em tempo de 15 dias e na concentração de $3 \%$ de i.a.

\section{CONCLUÇÕES}

Nas duas espécies de bambu, foi observada alta resistência ao ataque dos fungos xilófagos (Postia placenta e Polyporus fumosus) após os tratamentos preservativos. Contudo, as amostras que não receberam tratamento químico também foram resistentes aos ensaios de fungos, indicando que a constituição química, principalmente a presença de sílica, dos colmos pode ter influenciado no ensaio. Os colmos tratados com concentração de i.a. mais elevada e por maior período de tempo se mostraram mais resistentes.
Com base nas análises dos resultados de perda de massa dos ensaios de resistência ao ataque de fungos, pode-se concluir que o método de tratamento preservativo dos bambus por transpiração com o diafragma rompido submetido à solução com $3 \%$ de i.a. e em tempo de 15 dias foi o mais eficiente entre todos os tratamentos estudados.

Contudo, é necessário realizar algumas modificações nos métodos de tratamento preservativo para que seja alcançada penetração mínima recomendada pelas normas de tratamento de madeira.

\section{AGRADECIMENTOS}

Ao Conselho Nacional de Desenvolvimento Científico e Tecnológico (CNPq), pela Bolsa de Produtividade em Pesquisa (306398/2009-5) concedida ao segundo autor; à Fundação de Amparo à Pesquisa do Espírito Santo (FAPES), pela bolsa de Mestrado concedia ao primeiro autor; e à Montana Química S.A., pela doação do produto "MOQ OX 50", empregado no tratamento dos colmos de bambu.

\section{REFERÊNCIAS}

\section{AMERICAN SOCIETY FOR TESTING AND}

MATERIALS. ASTM D - 1413: standard test method for wood preservatives by laboratory soilblock cultures. Annual Book of ASTM Standard, Philadelphia: 2005a. 7p.

\section{AMERICAN SOCIETY FOR TESTING AND}

MATERIALS. ASTM D - 2017: standard method of accelerated laboratory test of natural decay resistance of wood. Annual Book of ASTM Standard, Philadelphia: 2005b. 5p.

\section{ASSOCIAÇÃO BRASILEIRA DE NORMAS}

TÉCNICAS. NBR 9480: peças roliças preservadas de eucalipto para construções rurais: requisitos. Rio de Janeiro, 2009. 12p.

BERALDO, A.L.; RIVERO, L.A. Bambu laminado colado (BLC). Floresta e Ambiente, v.10, n.2, p.36-46, 2003.

BERALDO, A.L.; SETTE, L.D.; BINATTI, M.F.; COSTA, R.B.S.; GOMES, R.S. Occurence of filamentous fungi on Dendrocalamus giganteus in Brazil. Disponível em: $<$ http:// www.apuama.org/admin/arquivos/pdfs/ bamboo_fungi.pdf>.Acesso em: 15 abr. 2012.

Revista Árvore, Viçosa-MG, v.39, n.3, p.567-574, 2015 
FARIAS SOBRINHO, D.W.; PAES, J.B.; FURTADO, D.A. Tratamento preservativo da madeira de algaroba (Prosopis juliflora (Sw.) D.C.), pelo método de substituição de seiva. Cerne, v.11, n.3, p.225-236, 2005.

HAOJIE, W.; VARMA, R.V.; TIANSEN, X. Insect pests of bamboos in Asia: an illustrated manual. New Delhi: International Network for Bamboo and Rattan, 1998. 200p.

HUI, C.; YANG, Y. China's bamboo: culture, resources, cultivation, utilization. 33. ed. Beijing: International Network for Bamboo and Rattan, 2010. 224p.

LIESE, W. Bamboos: biology, silvics, properties, utilization. Eschborn: Gesellschaft für Technische Zusammenarbeit - GTZ, 1985. 132p.

MOHANAN, C. Diseases of bamboos in Asia: an illustrated manual. New Delhi: International Network for Bamboo and Rattan, 1997.219p.

PAES, J.B. Viabilidade do tratamento preservativo de moirões de bracatinga (Mimosa scabrella Benth.), por meio de métodos simples, e comparações de sua tratabilidade com a do Eucalyptus viminalis Lab. 1991. 140f. Dissertação

(Mestrado em Engenharia Florestal) -

Universidade Federal do Paraná, Curitiba, 1991.

PAES, J.B.; MORESCHI, J.C.; LELLES, J.G. Avaliação do tratamento preservativo de moirões de Eucalyptus viminalis Lab. e de bracatinga (Mimosa scabrella Benth.) pelo método de substituição da seiva. Ciência Florestal, v.15, n.1, p.75-86, 2005.

PAES, J.B.; OLIVEIRA, A.K.F.; OLIVEIRA, E.; LIMA, C.R. Caracterização físico-mecânica do laminado colado de bambu (Dendrocalamus giganteus). Ciência Florestal, v.19, n.1, p.41$51,2009$.

SANTOS, R.L.; LOPES, J.D.S. Construções com bambu: opção de baixo custo. Viçosa, MG: Centro de Produções Técnica, 1998. 40p. (Série Construções Rurais, 160).

STEEL, R.G.D.; TORRIE, J.H. Principles and procedures of statistic: a biometrical approach. 2.ed. New York: Mc-Graw Hill, 1980. 633p.

SUPRAPTI, S. Decay resistance of five Indonesian bamboo species against fungi. Journal of Tropical Forest Science, v.22, n.3, p.287-294, 2010. 